\title{
Trayectorias: A new model for online task-based learning
}

\author{
CRISTINA ROS i SOLÉ and RAQUEL MARDOMINGO \\ Department of Languages, Faculty of Education and Language Studies, \\ The Open University, Milton Keynes MK7 6AA, UK \\ (e-mail: \{c.ros; r.mardomingo\}@open.ac.uk)
}

\begin{abstract}
This paper discusses a framework for designing online tasks that capitalizes on the possibilities that the Internet and the Web offer for language learning. To present such a framework, we draw from constructivist theories (Brooks \& Brooks, 1993) and their application to educational technology (Newby, Stepich, Lehman \& Russell, 1996; Jonassen, Mayes \& McAleese, 1993); second language learning and learning autonomy (Benson \& Voller, 1997); and distance education (Race, 1989; White, 1999). On the one hand our model balances the requirements of the need for control and learning autonomy by the independent language learner; and on the other, the possibilities that online task-based learning offer for new reading processes by taking into account new literacy models (Schetzer \& Warschauer, 2000), and the effect that the new media have on students' knowledge construction and understanding of texts. We explain how this model works in the design of reading tasks within the specific distance learning context of the Open University, UK. Trayectorias is a tool that consists of an open problem-solving Web-quest and provides students with 'scaffolding' that guides their navigation around the Web whilst modelling learning approaches and new learning paradigms triggered by the medium. We then discuss a small-scale trial with a cohort of students $(\mathrm{n}=$ 23). This trial had a double purpose: (a) to evaluate to what extent the writing task fulfilled the investigators' intentions; and (b) to obtain some information about the students' perceptions of the task.
\end{abstract}

\section{Introduction}

In the last few decades we have witnessed a growing interest in the use of the Web as a source for authentic materials and for reading in the context of language learning. Indeed, until recently, the Web was mainly viewed as a great bank of information that followed static models for the transmission of knowledge. Current views, however, see the Web as the means to engage in more dynamic and interactive processes. In this paper we will argue that applications for L2 online reading need to adopt new paradigms that take this new vision of the Web into account.

Although the exploration of how learners read online has received a great deal of attention in the last few years (Chun, 2001; Lomicka, 1998; Ward \& Newlands, 1998), few researchers have addressed the need to look at task design and reading processes 
that take into account new literacy models, and the effect that new media has on students' knowledge construction and understanding of texts (Richards, 2000, Schetzer \& Warschauer, 2000; Kramsch \& Andersen, 1999). To achieve this, new insights from task-based learning, constructivist education, learning autonomy, and new literacies need to be applied to the design of a new model for online task-based learning.

Constructivism and socio-cultural approaches to learning have been hailed as the most useful learning theories for networked environments, giving learners increasingly prominent roles for interpreting meaning. Similarly, task-based learning has become one of the most accepted modes of instruction in the last few decades due to its suitability to both communicative and constructivist approaches to learning. Moreover, the flexibility and open-ended format of a task lends itself to the hypermedia nature of the Web and its application to online learning. Up until recently, online task design has tended to follow static models of the Web and the Internet in general, where the interaction and collaboration that takes place is off-line rather than online (e.g. Cabot, 2000; Gánem-Gutiérrez, 2003; González-Lloret, 2003).

Lastly, the need for greater levels of autonomy by distance language learning and students generally engaging in self-study, prompt us to reflect on what characteristics a task should have to accommodate and promote students' self-awareness of the learning process.

Next, we will describe the theoretical underpinnings for this model, explaining and illustrating how they have been implemented in the task.

\section{Theoretical background}

\subsection{Task-based learning}

The task-based learning theory discussed here has been drawn from discussions put forward by Willis (1996) and Ellis (2000) for traditional print medium in a face-to-face context. To this we add what the Web-based medium and online context can contribute to task design.

\subsubsection{Face-to-face task-based design}

One of the main proponents of task-based learning defines tasks as 'Activities where the target language is used by the learner for a communicative purpose (goal) in order to achieve an outcome' (Willis 1996:23). In other words, there are two crucial characteristics of a task, its focus on meaning and communicative value, and the goal and objective of the task.

An important addition to Willis's definition (1996) and to those of earlier theorists (e.g. Prabhu, 1987) is Skehan's (1998a in Ellis, 2000) requirement that the task resembles the real world. This is a key element if the aim is that students interact with and within the target culture. Equally important is Ellis's (2000) concept of task 'orientation'. Tasks, according to Ellis, practise linguistic skills through the task itself rather than assuming linguistic ability as a pre-requisite for the task, which means that the orientation of the task is essentially towards the content rather than towards the linguistic structures to be acquired. Ellis also points out that this goal can be achieved in different ways, as the learning that takes place in a task should allow for improvisation and discovery. 


\subsection{2 online task types}

Although there have been many classifications of tasks in the face-to-face context, these become insufficient when we try to describe the range and type of language activities available online. New communication tools and text types, different types of media, which allow for multimodal texts (Kress \& Van Leeuwen, 2001), also require new learning strategies and, even, new skills. Indeed, electronic tasks require new instructional paradigms that reflect the type of learning that takes place online.

Based on her extensive knowledge of existing tasks for the Web, Felix (2002a) distinguishes two main types of Web-based activities:

- Information-gap resolution models, which include mystery games, contextualised language and research tasks embedded in Web-quests (with and without problem-solving element) and creative simulations. These are mostly designed, produced and monitored by teachers rather than learners.

- Experiential learning models, which emphasize students' active involvement in negotiating the curriculum and shaping both the process and the outcome of the learning.

According to Felix (2002b), Web-quests (information-gap resolution models) are one of the most widespread Web-based activity types. A Web-quest consists of a task where students have to access relevant Websites in order to compile specific information on a topic, and give answers to questions. We believe, however, that the two categories described above are not enough for providing a model for online task-based learning as they do not allow the learner to get involved in directing her own learning.

Instead, we propose to establish a continuum of task types where we can distinguish a gradual progression from teacher centered information-gap resolution models, to less teacher centered or experiential learning models. From our point of view, the type of Web-quest with a problem-solving task that Felix (2002b) proposes could lend itself to a diversity of treatments, not only one that is controlled by the teacher. This would give a different degree of autonomy and control to the learner, depending on the openness of the task. Web-quests with a problem-solving element are optimum task environments for a more flexible learning, and would be susceptible to being placed in between the Information-gap resolution and the Experiential learning model, as illustrated in Figure 1. As well as being a flexible model for task-based online learning, this new view is grounded on current theories for online learning. Below, we expand on these theories and explain how they contribute to the model.

\subsection{Constructivism}

Constructivist approaches to learning have been gaining ground in educational technology paradigms in higher education contexts for the last decade. The focus of learning is radically changed and places the emphasis on how the individual interacts with the world rather than focusing on the internal mechanisms for acquiring information. Learners need to take centre stage in the learning process by following their own ideas and goals whilst actively involving themselves in knowledge construction (Brooks \& 


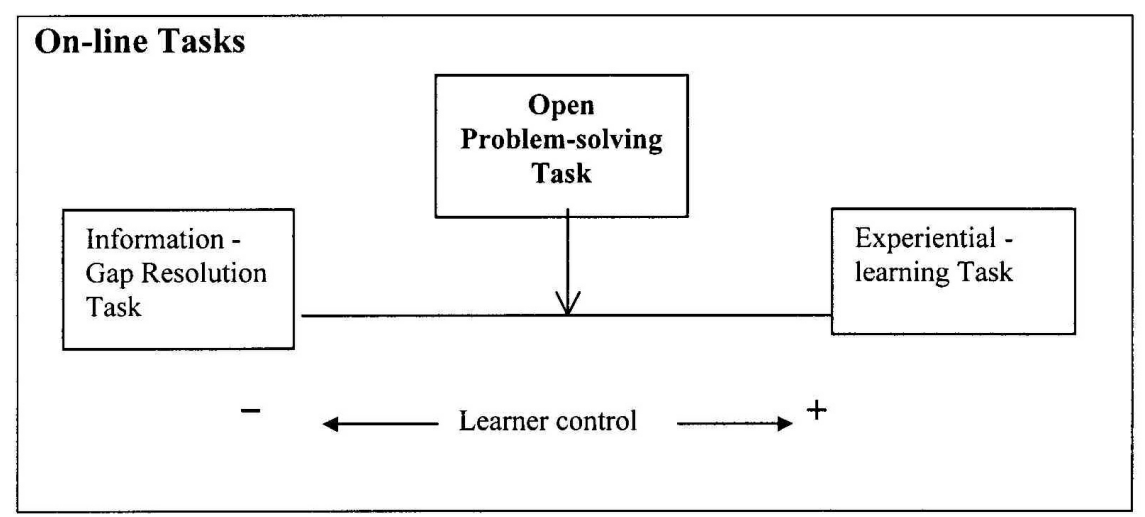

Fig. 1. Pedagogical model for online tasks.

Brooks, 1993; Jonassen et al., 1993; Felix, 2002a). In constructivist online learning, learners manipulate new information and form their own opinions by reconstructing their knowledge continuously (Jonassen et al., 1993; Newby et al., 1996).

According to constructivist online task design, tasks are set in contexts, so that knowledge and the conditions in which this knowledge occurs are inextricably bound together (Felix, 2002a). These contexts should be rich learning environments and situations, which present a world that is complex, has multiple perspectives, and where information can be contradictory. To achieve this, tasks should present different sources and resources while promoting exploration (Rüschoff, 2002; Newby et al., 1996).

As well as being aware of their individual learning processes, learners need to go beyond themselves and engage in collaboration, either with other students, with a teacher who provides guidance, or by the collaboration that it is established between the different texts that the reader visits in a 'to and fro' fashion (Shetzer \& Warshauer, 2000).

The next section further explains why learners need to become self-aware and how such self-awareness can be promoted in online task-based learning.

\subsection{Autonomy and distance learning}

When designing tasks for distance online learning, the role played by the development of autonomy in the learning process cannot be underestimated. Understanding the learning objectives and taking responsibility for them are two of the most important areas that learners will have to address in order to become autonomous (Little, 2001). To achieve this degree of autonomy, learners have to acquire increasingly more control of their own learning.

This is particularly true for the distance learning context, where it is important to develop students as learners by making them aware of their own goals and abilities. According to Benson (1997), once learners identify the pedagogical goals of the materials they are using, they have achieved the first steps to able to identify their own preferred learning style and strategies. Fostering self-awareness, therefore, in the form of 
learner training, learning strategies and personalized feedback, is an important aspect in the design of an online task.

Lastly, motivation is a crucial factor in independent online learning. Theorists of distance language learning highlight the importance of this factor (Race, 1989; White, 1999). And as Hurd, Beaven and Ortega (2001:342) say 'motivation is essential, as with it comes persistance when the going gets tough'.

The next section provides a brief overview of the new skills that are developed in online tasks.

\subsection{New literacies}

We have to acknowledge that online tasks require new learning models. This is partly because online texts are intrinsically different from print texts. Some of the most striking characteristics of this medium have already been identified, e.g. nonlinearity, where the student can follow her preferred learning path and order of exploring the materials, and the multi-modality of texts.

This new type of reading is also inextricably bound up with creating knowledge and critical judgment. As Richards (2000) states, the most fundamental difference when reading on the Internet is the changing role of the reader, from a passive to an active one. In fact, it is only recently that it has been recognized that the new electronic media and the Internet involve interactive, critical and agency processes - the processes by which readers create new texts from their personal interpretations of the reading. Synder (1997 in Richards, 2000:62) believes that in hypertext, text is partly constructed by the writers and partly by the readers who decide what links to pursue.

As Warschauer (2001) points out, some of the new skills that this type of reader will need to master are 'research skills', which can consist of 'searching', 'selecting' and 'evaluating' what one finds, judging validity/reliability and accuracy of information and making decisions on what links to pursue. This warrants the claim that reading on the Web should follow new models of literacy, which include new hybrid skills. Users and learners are not only reading texts online but by virtue of making their own choices and interpreting them through their own experience they can also create and 'write' a different text.

To conclude this section we summarise the different elements that need to be taken into account when designing an online task for reading purposes.

1. have an orientation towards meaning

2. state learning goals clearly

3. promote exploration, improvisation and discovery

4. motivate by presenting real, complex settings and world relationship

5. get the learner to be personally involved

6. include a problem-solving element

7. help the learner to become self-aware

8. allow the learner to become an active reader.

In the next section we explain how the main principles of an online task-based model, as described above, were applied to the Trayectorias task. 


\section{Applying the model to the distance learning context}

\subsection{Introducing online technologies to the curriculum}

The task we are going to describe has been designed with Open University students in mind; these students follow their studies at home with tutor support. In order to do this, students have at their disposal a wealth of print, electronic and online materials. Recently, there has been a move towards a higher integration of new learning technologies into the curriculum by gradually extending the provision of electronic resources and online language tuition. This involves both the use of synchronous and asynchronous communication tools (see Hampel, 2003; Hauck \& Haezewindt, 1999; Lamy \& Goodfellow, 1999; Lamy \& Hassan, 2003; Shield \& Hewer, 1999), and an e-desktop environment that allows students to access linguistic resources and create online communities (Álvarez \& Beaven, 2002).

One of the resources available to students is a purpose-built e-desktop. As well as giving access to information about the course, this provides students with a selected list of course-related URLs. Although there are no integrated tasks in the curriculum that are exclusively carried out with Web resources at present, it was assumed that using the Web to research different cultural topics or look for information are routine exercises that learners engage in whilst studying the foreign language. Our objective, therefore, was to investigate how the student goes about researching a topic online and how to support this with a task that guides these searches. The Trayectorias task helps students to make the most of the online resources available to them and to analyse these, relate the information to their knowledge, and construct their own interpretation of a particular topic.

\subsection{The Trayectorias design}

Trayectorias is an online task which uses pre-selected live authentic Web pages to present a particular historical period from the Hispanic world. In order to participate in the task, the student is asked to follow the "trajectories" of nine characters from that time, namely, some of the main figures from the historical period immediately before and after the Second World War in different Spanish speaking countries on both sides of the Atlantic. The student has to navigate through the media-rich pages to find the points of contact between the different personalities and thus construct a personal vision of the historical period.

This task was designed on the Web using HTML and provides scaffolding to help the learner to navigate through the information. Students also have the choice of following up links they might be interested in and are also encouraged to look for other appropriate Web pages if they wish to.

Trayectorias can be completed in three different ways, depending on which route the student chooses. In Ruta 1, the student decides how she wishes to structure her search according to her own preferences and motivation. In Ruta 2, the student is given a subtask to structure her work, which consists of making comparisons and choosing a topic. Finally, in Ruta 3, the student is given another intermediate sub-task, which consists of a series of questions, on specific pieces of information about the characters' lives.

In all three routes, the students have access to a commercial online dictionary as well 
as to a help facility in English. The latter feature explains how to navigate through the pages of Trayectorias and gives some clues, in case users experience any difficulties with the activity. Students can also obtain help in terms of their linguistic performance by getting a model answer, which shows how they could have responded to the task, and a strategy training session developed by the teachers/investigators with tips for reading online and autonomous learning that students can access at any time.

\subsection{Applying the model for online tasks to Trayectorias}

In this section we explain how the theoretical model for online task design applies to our Trayectorias task.

i. 'Having an orientation towards meaning': to orientate the learner towards the task, the student is introduced to the topic, the historical period before and shortly after the Second World War in the Hispanic world. After this introduction (illustrated in Figure 2), she begins to explore the lives of the different personalities and how these are a reflection of the times. As a result, the linguistic structures will be practised through the thematic work, rather than the other way around.

ii. 'State learning goals clearly': the learner is provided with a clear goal for her task: to write a short paragraph about her appreciation of the historical period she has worked on. In this way, whatever Ruta (route) the student takes, she has a plan, and a single goal for learning.

iii. 'Promote exploration, discovery and improvisation': the student is expected to explore the different sources of information on the selected Web pages, but she is also encouraged to search for her own resources. To promote discovery and improvisation, the student is given the choice to follow one of the three routes to complete the task. This will not determine the type of exploration each student will do, as whatever route is taken, the task will always allow for discovery and a context-dependent approach to learning.

iv. 'Motivate by presenting real and complex settings, and world relationships': the topics presented are real characters of the Hispanic speaking world who have been selected both to appeal to students' background knowledge and to reflect the real historical setting of the period, with its complexities and contradictions. Students are presented with characters with whom they are likely to be already acquainted, e.g. Eva Perón, Federico García Lorca; and others, such as María Félix and Pau Casals, who will be less known to them. All these characters represent different aspects and perspectives on this historical period (see Figure 4).

v. 'Get the learner to be personally involved': in Trayectorias, the student is the creator of meaning and she constructs her personal image of the Hispanic speaking culture of the time by bringing her previous experience to the interpretation of the historical period.

vi. 'Include a problem-solving task': Trayectorias is a Web-quest with an open problem-solving goal. This task fulfils the requirement described above of going beyond the information-gap resolution model task. Although students need to gather information from different Web sites to construct their answer, they also need to interpret 


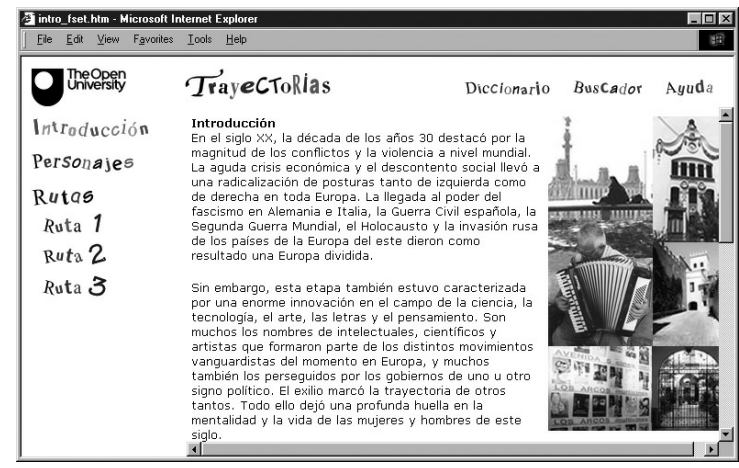

Fig. 2. Orientation on meaning: Task's introduction to the historical context.

and link the information to find the points of contact between the different characters, and use summary skills. Moreover, the task does not have a right or wrong answer, rather, it prompts different outcomes from different students.

vii. 'Help the learner become self-aware': to make students become more aware of how, what and why they learn, and to take increasing control of and responsibility for their learning, the authors made an audio-recording that provides advice on how to learn and the use of learning strategies. In order to promote reflection about learning styles and processes, there are also three different instructional styles embedded in the task: serialistic (Ruta 3), holistic (Ruta 1), or mixed (Ruta 2 ), which is a combination of the two. The serialistic approach looks at the materials in a hierarchical manner, step by step, whereas the holistic approach has a more unstructured way of dealing with the text in a more exploratory manner, where knowledge is 'grazed' from the text (Pask, 1976, in Jones et al.,1997).

viii. 'Allow the learner to become an active reader'. The task not only poses some challenges to the student from the point of view of her cultural knowledge, but also presents complexity by requiring the learner to be more active, i.e. by selecting the characters she concentrates on, making decisions about which links to

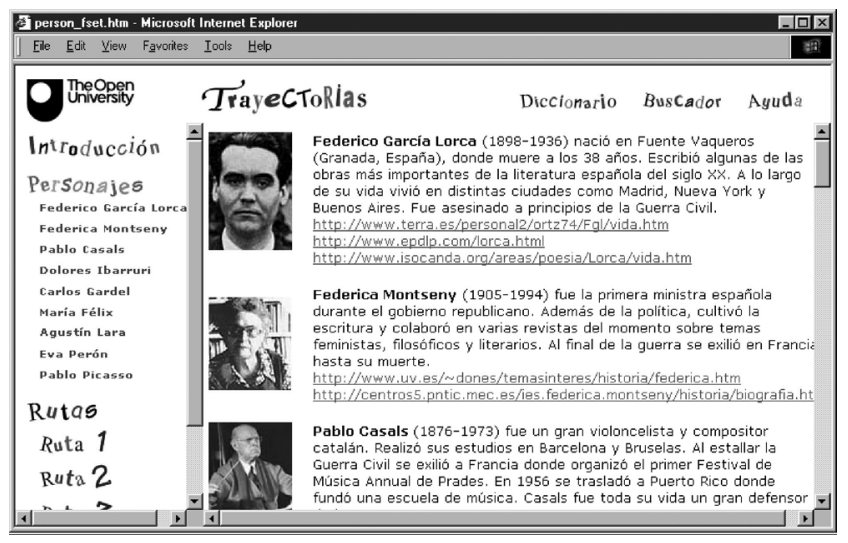

Fig. 3. Presenting real and complex settings. 


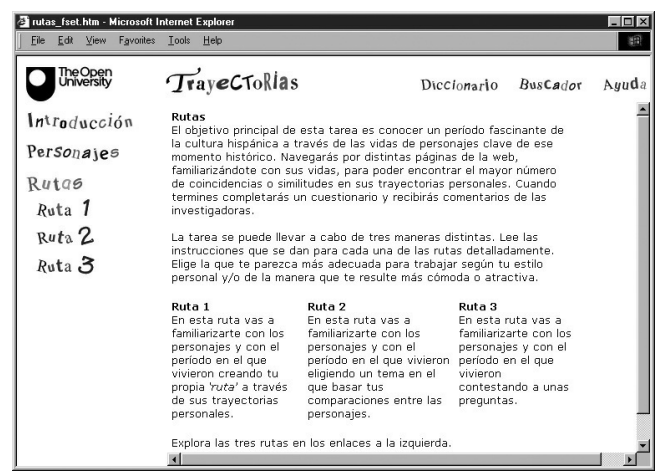

Fig. 4. Help the learner become self-aware: choice of instructional styles.

follow, relating the information within these, and interpreting it according to her interests and mental structures.

\section{Evaluation}

This task has undergone two evaluations. The first one was a pilot study conducted with the first version of the tool (Ros i Sole et al., 2001). The results of that investigation indicated that although students enjoyed the task, they did not seem to make use of its full potential, as they preferred one of the three routes. Namely, they perceived the first route as being the easiest and more secure of the three. To make sure that perceived difficulty or other variables did not affect their choice, we made a series of modifications to the design:

- Inversion of the order of the three routes. We placed the previously least popular route in the first position.

- Omission of routes' title to avoid any interpretation that one route was easier or better than any other.

- Addition of scaffolding so that the instructions gave the same level of support for each route.

- Clarification of common learning outcome.

- Location of a live version of Trayectorias on the Web, so that the task would be more similar to a real-world Web-based task.

- Addition of an autonomy and strategy training session to provide greater focus on reading strategies and learning autonomy on the Web.

In the following section we shall explain how the second version of the Trayectorias tool, the one described in this paper, was evaluated. A first check was done for functionality purposes. All the tools and options built into the model were tried and its functionality tested. Subsequently, a small-scale trial was conducted with a cohort of students (n $=23$ ). This trial had a double purpose, (a) to evaluate to what extent the writing task fulfilled the investigators' intentions - this was done through a brief linguistic analysis of the texts produced by students; (b) to obtain some information about the students' perceptions of the task, which was carried out using an online questionnaire. 


\subsection{Methodology}

The tool has been trialled with students from two different Higher Education institutions in the UK (The Open University and Leeds University) who were taking Spanish as part of their undergraduate studies. They both had a similar level of language proficiency. The majority of these students were English native speakers and their language level was intermediate. Most also reported being 'fairly familiar' or 'very familiar' with computers, the Internet and the Web. Students were asked to complete the task and a questionnaire and send it to the researchers. The analysis of their tasks was used to measure one particular element of the task design, student personal involvement. The questionnaire, on the other hand, was designed to canvass the general perception of students on the task and their perceived failures and successes of the task. The questionnaire was built into the task and students could only access it at the end of it. The completed questionnaire, therefore, was sent to the researchers at the same time as their completed language task.

\subsection{Data from students' work}

We are next going to discuss the results of students' work on the task, and in particular, their personal involvement. Table 1 shows extracts where students use different ways of introducing their descriptions of the historical period. We analysed some of these phrases by looking for signs of personal involvement in the task. The answers reflect that their writings go beyond objective accounts of what they have read, and that students filter the information through their personal experience. In the first case, student 1 explicitly shows that the answer she is about to give is her personal opinion. In student 2 , we see that there is some tentativeness in the answer, which is expressed by the use of the phrase 'they can' (se pueden), which signals that she acknowledges the subjectivity of the answer. In student 3, we see that the answer is being justified by the student telling us about what kind of person she is and, therefore, admitting that this is one of many outcomes of the task.

This brief analysis gives us an indication that students were using the task as intended, that is to say, not as a mere Web-quest, or a tool for gathering information, but as a tool to explore new online resources, that allowed them to solve a problem, and to create individual responses to texts.

\subsection{Questionnaire feedback}

The results of the questionnaire reinforce the view that the application of the online Task model to the design of Trayectorias has achieved its intended objectives. Some relevant data from the questionnaire is shown in the list below.

- Meaning-focussed: the content - personalities and historical period - was reported to be the most enjoyable aspect of the task.

- Improvisation and choice. More than half chose the route that fitted best with the way they learn. Almost all made an informed choice of route (i.e. they read the other routes before choosing). Over half preferred route 3 . 
Table 1 Students' different use of introducing a description of a historical period

\begin{tabular}{lll}
\hline \hline Student identification & Spanish phrase used & Translation \\
\hline 1 & Para mi... & $\begin{array}{l}\text { In my opinion... } \\
\text { The characters can be } \\
\text { grouped in... }\end{array}$ \\
3 & $\begin{array}{l}\text { Los personajes se pueden agrupar... } \\
\text { Considero a mi persona siendo una } \\
\text { que tiene un espíritu artístico y } \\
\text { humorístico... }\end{array}$ & \\
\hline \hline
\end{tabular}

- Self-awareness of learning processes. Over half said the task helped to improve on reading strategies and to develop learning autonomy. Half agreed that choosing a route helped them to reflect on problem-solving strategies.

As we see from these results, the students felt that the emphasis of the task was on content, they had enough freedom to navigate through the materials and make their own choices about what topics to pursue, even though the preferred route was still the one that allowed for less discovery learning (Ruta 3). Moreover, most of them felt that the task helped them to become more aware of their learning processes by giving them the choice of different instructional styles and by helping them to reflect on the use of their own strategies. One issue that stands out from the questionnaire, however, is that learners were still overwhelmed by the amount of information that performing a reading task with multimodal and hyperlinked texts provided them with, and the infinite possibilities for discovery and improvisation that this afforded.

\section{Conclusion}

Task-based learning is the pedagogical approach that has provided us with a flexible enough framework for the learner to be actively involved in the learning. Similarly, a constructivist approach has helped to deepen our understanding of what type of learning occurs when the learner's knowledge takes center-stage, and by requiring that tasks be set in rich learning environments in which students engage with the texts and reconstruct their previous knowledge.

Although it has been stated that online tasks should follow more experiential modes of learning, it is also true that these types of tasks require students to develop a high level of learning autonomy and self-awareness. We have also seen that new literacy skills entail new learning processes that warrant the need for new reading strategy training. Indeed, designing online tasks for distance or independent learning contexts cannot be approached from purely experiential learning models, as not all learners will be prepared to assume the level of control and personal involvement required by this model, as our brief evaluation shows.

On the contrary, when learners approach an online reading task, they need to be provided with a plan, or scaffolding that guides them through their searches and constructions of meaning. This type of support and guidance is provided by our online model task. Trayectorias has provided such a framework by offering both an element of 
planning and of improvisation so that learners at different levels of autonomy can be successful at completing the task independently.

Although learners will gradually acquire higher degrees of self-awareness to venture into more experiential routes and interactive environments, we need to offer a flexible enough model so that less independent learners can also take part in the construction of their own learning experience with online reading tasks.

\section{Acknowledgements}

We would like to thank Ali Wyllie, Joan-Tomàs Pujolà, Manuel Frutos-Pérez, and Antonio Martínez-Arboleda for their contribution at different stages of this project, and Regine Hampel and Lesley Shield for their helpful comments on earlier drafts of this article. We would also like to thank the OU Research Development Fund and the Department of Languages at the Open University for supporting this research project.

\section{References}

Álvarez, I. and Beaven, T. (2002) Developing intercultural competence for the knowledge society: The Open University A buen puerto Website. Proceedings of Setting the Agenda Conference, Manchester, 2002. http:www.lang.ltsn.ac.uk/resources/conference2002.aspx

Benson, P. (1997) The philosophy and politics of learner autonomy. In: Benson, P. and Voller, P. (eds.), Autonomy and Independence in Language Learning. Longman: Harlow.

Benson, P. and Voller, P. (1997) Autonomy and Independence in Language Learning. Longman: Harlow.

Brooks, J. G. and Brooks, M. G. (1993) The Case for Constructivist Classrooms. Alexandria, Va.:Association for Supervision and Curriculum Development.

Cabot, C. (2000) The effects of the World Wide Web on reading and writing skills in a Spanish cultural studies course. ReCALL 12(1): 63-72.

Chun, D. M. (2001) L2 Reading on the Web: strategies for accessing information in hypermedia. Computer Assisted Language Learning 14(5): 367-403.

Ellis, R. (2000) Task-based research and language pedagogy. Language Teaching Research 4(3): 193-220.

Felix, U. (2002a) The Web as a vehicle for constructivist approaches in language teaching. ReCALL 14(1): 2-15.

Felix, U. (2002b) Schritt halten mit dem Web: die Aufgabe lösen. http://www.gfl-journal.de/12002/felix.html.

González-Lloret, M. (2003) Designing task-based Call to promote interaction: En Busca de Esmeraldas. Language Learning and Technology 7(1): 86-104.

Gánem-Gutiérrez, A. (2003) Beyond interaction: the study of collaborative activity in computermediated tasks. ReCALL 15(1): 94-112.

Hampel, R. (2003) Theoretical perspectives and new practices in audio-graphic conferencing for language learning. ReCALL 15(1): 21-36.

Hauck, M. and Haezewindt, B. (1999) Addding a new perspective to distance (language) learning and teaching -the tutor's perspective. ReCALL 11(2): 46-54.

Hurd, S., Beaven, T. and Ortega, A. (2001) Developing autonomy in a distance language learning context: issues and dilemmas for course writers. System 29: 341-355.

Jonassen, D. H., Mayes, T. and McAleese, R. (1993) A manifesto for a constructivist approach to uses of technology in higher education. In: Designing Environments for Constructive Learning. 
London: Springer-Verlag, 231-247.

Jones, P., Jacobs, G. and Brown, S. (1997) Learning styles and CAL design: a model for the future. Active Learning 7: 9-13.

Kramsch, C. and Andersen, R. W. (1999) Teaching text and context through multimedia. Language Learning and Technology 2(2): 31-42.

Kress, G. and Van Leeuwen, T. (2001) Multimodal Discourse: The modes and media of contemporary communication. London: Arnold.

Lamy, M. and Goodfellow, R. (1999) Supporting language students' interactions in Web-based conferencing. Computer Assisted Language Learning 12(5): 457-477.

Lamy, M.-N. and Hassan, X. P. (2003) What influences reflective interaction in distance peer learning? Evidence from four long-term online learners of French. Open Learning 18(1): 39-59.

Little, D. (2002) Learner autonomy and second/foreign language learning. In: The Guide to Good Practice for learning and teaching in Languages, Linguistics and Area Studies. LTSN Subject Centre for Languages, Linguistics and Area Studies, University of Southampton.

Lomicka, L. L. (1998) To gloss or not to gloss: an investigation of reading comprehension online. Language Learning 1(2): 41-50.

Newby, J. N., Stepich, D. A., Lehman, J. D. and Russell, J. D. (1996) Theory into application. In: Instructional Technology for Teaching and Learning: Designing Instruction, Integrating Computers, and using Media. New Jersey, USA: Merrill/Prentice Hall, 25-34.

Race, P. (1989) The Open Learning Handbook: Selecting, Designing and Supporting Learning Materials. London: Kogan Page.

Richards, C. (2000) Hypermedia, Internet communication, and the challenge of redefining literacy in the electronic age. Language Learning and Technology 4(2): 59-77.

Prabhu, N. S. (1987). Second Language Pedagogy. Oxford: OUP.

Ros i Solé, C., Mardomingo, R. and Pujolà, J. (2001) Trayectorias: Una tarea para fomentar la autonomía del aprendizaje en la red. In: Tecnologías de la Información y de las Comunicaciones en la enseñanza de ELE. Proceedings from ASELE, Valencia: Universidad Politécnica de Valencia.

Rüschoff, B. (2002) Construction of knowledge as the basis of foreign language learning. http://www.uni-essen.de/anglistik/construction; http://www.uni-essen.de/anglistik/bernd/construction.htm (last accessed 21.05.03).

Schetzer, H. and Warschauer, M.(2000). An electronic literacy approach to networked-based language teaching. In: Warschauer, M. and Kern, R. (eds.), Networked-Based Language Teaching: Concepts and Practice. New York: CUP.

Shield, L. and Hewer, S. (1999) A synchronous learning environment to support distance language learners. In: Cameron, K. (ed.), CALL and the Learning Community. Exeter: Elm Bank Publications.

Trayectorias. http://trayectorias.open.ac.uk

Ward, M. and Newlands, D. (1998) Use of the Web in undergraduate teaching. Computers and Education 31: 171-184.

Warschauer, M. (2001) The death of cyberspace and the rebirth of CALL. In: CALL in the 21st Century. CD-ROM. IATFL.

White, C. (1999) Expectations and emergent beliefs of self-instructed language learners. System 27: 443-457.

Willis, J. (1996) A Framework for Task Based Learning. Harlow: Longman. 